\title{
Postural Stabilization of Visually Guided Eye Movements
}

\author{
Thomas A. Stoffregen \\ School of Kinesiology \\ University of Minnesota \\ Benoît G. Bardy \\ Faculty of Sport and Movement Sciences \\ Université Montpellier 1 and Institut Universitaire de France \\ Cedrick T. Bonnet \\ School of Kinesiology \\ University of Minnesota \\ Randy J. Pagulayan \\ Department of Psychology \\ University of Cincinnati, Cincinnati, Ohio
}

\begin{abstract}
We studied relations between eye movements and postural control. In two experiments, participants were asked to shift gaze to follow horizontal oscillation of visual targets. Postural sway variability was reduced during target oscillation, relative to sway with a stationary target. Target displacement amplitude was within the range that normally does not elicit head rotation, and measured head rotation did not increase during target motion. Eye movements made when the eyes were closed did not yield a reduction in body sway (relative to sway when the closed eyes were stationary). The amplitude and frequency of eye movements matched the amplitude and frequency of target motion. The results undermine the view that eye movements and postural control compete for limited central processing resources, and document a functional integration of postural control with visual performance.
\end{abstract}

Tasks or behavioral goals that are superordinate to the control of posture may be termed suprapostural tasks (Stoffregen, Pagulayan, Bardy, \& Hettinger, 2000;

Correspondence should be addressed to Thomas A. Stoffregen, School of Kinesiology, 202B Cooke Hall, University of Minnesota, Minneapolis, MN 55455. E-mail: tas@umn.edu. 
Stoffregen, Smart, Bardy, \& Pagulayan, 1999). Suprapostural tasks and postural control are defined and evaluated in qualitatively different terms. For example, reading is not evaluated in terms of the position or motion of the body's center of mass, the minimization of global optical flow, and so on. Body sway can influence performance at some suprapostural tasks (e.g., uncontrolled sway can interfere with reading), but performance of suprapostural tasks is measured in different terms (e.g., reading rate or comprehension). In general, values of postural parameters are not informative about suprapostural task performance. For example, a large excursion of the center of mass might degrade visual performance (e.g., if the person began to fall over), but it could facilitate visual performance (e.g., if the person leaned to bring the eyes closer to a visual target).

It is widely understood that suprapostural activity can influence the control of stance (e.g., Bernstein, 1996; Jeka, 1995; Newell, 1986; Reed, 1988; Slijper \& Latash, 2000; Woollacott \& Shumway-Cook, 2002). This effect typically is attributed to one of two causes. Many suprapostural activities involve motions of the body or its parts such as occur in reaching, walking, and so on. In such cases, suprapostural activity will influence posture because these suprapostural activities tend to affect the position or motion of the center of mass (e.g., Commissaris \& Toussaint, 1997; Friedli, Hallett, \& Simon, 1984; Lee, 1980; Slijper \& Latash, 2000). Perturbations of the center of mass are thought to have a direct influence on postural control (e.g., Giese, Dijkstra, Schöner, \& Gielen, 1996; Nashner \& McCollum, 1985).

Posture can be influenced even when suprapostural activity does not perturb the center of mass. Postural control is influenced by tasks, such as visual search, verbal reaction time, and mental arithmetic, that appear to have no mechanical influence on the position or motion of the center of mass (e.g., Dault, Geurts, Mulder, \& Duygens, 2001; Hunter \& Hoffman, 2001; Kerr, Condon, \& McDonald, 1985; Maylor \& Wing, 1996; Stoffregen et al., 2000; Stoffregen et al., 1999; Teasdale, Bard, LaRue, \& Fleury, 1993). Effects of this kind have often been interpreted in terms of cognitive processes such as the allocation of central processing resources (for a review, see Woollacott \& Shumway-Cook, 2002). An alternative interpretation is that posture might be controlled, in part, so as to facilitate performance of suprapostural tasks that make demands on perceptual performance (Riccio \& Stoffregen, 1988), such as touching (Riley, Stoffregen, Grocki, \& Turvey, 1999) and looking (Stoffregen et al., 2000; Stoffregen et al., 1999). In this study we evaluated the hypothesis that postural control might be modulated adaptively to facilitate changes in the direction of gaze. Our results suggest that postural control actions can be tuned to facilitate the control of changes in the direction of gaze.

In this article we distinguish between saccadic eye movements that are visually guided (i.e., saccades whose success is evaluated in terms of their tendency to maintain or improve the visibility of objects and events in the environment) and those that do not depend on visual guidance (e.g., saccades made in the dark or with the eyes closed). Visually guided eye movements are used to change the direction of gaze or to shift gaze. Gaze shifts can be accomplished by visually guided eye 
movements, by visually guided head movements (rotations), or by a combination of these. When the angular change in the direction of gaze is less than $15^{\circ}$, shifts in gaze commonly are achieved without rotation of the head, using only visually guided saccades (Hallet, 1986). In our experiments experimentally induced shifts in gaze were always less than $15^{\circ}$. For this reason, in this article the terms changes in the direction of gaze, shifts in gaze, and visually guided eye movements are equivalent.

\section{CENTRAL COMPETITION OR FUNCTIONAL INTEGRATION?}

A substantial literature has grown up around the idea that there may be important relations between the control of posture and the execution of simultaneous suprapostural tasks. In the typical paradigm, experimenters measure body sway while standing participants perform a suprapostural task. Sway under these conditions is compared with sway observed during "quiet stance," that is, without an explicit suprapostural task. Several studies have reported significant increases in body sway during performance of an explicit suprapostural task, such as visual matching (Dault et al., 2001), auditory reaction time (e.g., Lajoie, Teasdale, Bard, \& Fleury, 1993), or mental arithmetic (e.g., Maylor \& Wing, 1996; Yardley, Gardner, Leadbetter, \& Lavie, 1999). Researchers have concentrated on understanding the increase in body sway that sometimes accompanies tasks of this kind. These effects have often been interpreted in terms of the competitive allocation of central processing resources to postural and suprapostural activities (e.g., Lajoie et al., 1993; Teasdale et al., 1993) and more generally, between two tasks being performed simultaneously (e.g., Abernethy, 1988; Navon \& Gopher, 1979). The general idea is that postural and suprapostural activities constitute separate tasks, each of which draws on a central pool of cognitive processing resources. This view leads to the general prediction that combination of postural control with suprapostural activity should lead to degradation in performance of postural control, of the suprapostural task, or both.

By contrast, we have argued that postural control can be tuned to support the achievement of suprapostural activities (Riccio \& Stoffregen, 1988; Riley, Mitra, Stoffregen, \& Turvey, 1997; Riley et al., 1999; Stoffregen et al., 2000; Stoffregen et al., 1999). Our view suggests that, rather than interacting in a competitive manner, posture and certain types of suprapostural activity can be integrated so that their simultaneous performance need not lead to degradation in either and can, in fact, lead to improved performance. In the next section, we review the empirical basis for this view.

\section{Postural Stabilization of Visual Performance}

Stoffregen et al. (2000) measured postural sway while standing participants performed one of two visual tasks. One group of participants looked at a blank target 
(participants were not asked to maintain fixation on a specific point, and so we refer to this as an inspection task, not a fixation task). A second group of participants looked at a target covered with text, and were asked to search the text, identifying and counting instances of a designated target letter. The manipulation of visual task was crossed with variation in the distance of targets (the visual angle of targets was held constant across variations in distance). The amplitude of postural sway was operationalized in terms of the standard deviation of body position. There was a main effect of suprapostural task on the variability of sway (there was also a main effect of target distance; cf. Stoffregen et al., 1999). As predicted, during visual search, the variability of anterior-posterior (AP) sway was significantly lower than during inspection of the blank targets.

The visual search task used by Stoffregen et al. (2000) required an extended series of coordinated shifts of gaze (visually guided eye movements), alternating with brief periods of stationary gaze (fixation). In contrast, the inspection task was tolerant of reduced precision in the control of shifting and stationary gaze. Functional changes in sway might have been organized to facilitate the control of stationary gaze, of shifting gaze, or both. In this study, we evaluated the hypothesis that body sway can be modulated to support changes in the direction of gaze. Following our earlier research (Riccio \& Stoffregen, 1988; Riley et al., 1997; Riley et al., 1999; Stoffregen et al., 2000; Stoffregen et al., 1999), we hypothesized that there would be a functional relationship between body sway and visually guided eye movements. This is because the accuracy of such movements can be influenced by small changes in body sway. By contrast, eye movements that are not visually guided (e.g., eye movements made in the dark, or with eyes closed) are not subject to facilitation or disruption by small changes in body sway.

\section{Eye Movements and Body Sway}

The finding that body sway is reduced during a visual search task (Stoffregen et al., 2000) raises the possibility of a functional relation between body sway and eye movements: During visual search, body sway might be modulated to facilitate eye movements that shift gaze. Very little existing research is relevant to this issue. There is a large literature on relations between vision and posture (for partial reviews, see Warren, 1995, 1998), and there is a large literature on eye movements (for a review, see Rayner, 1998), but the overlap between these literatures is small. This is because the majority of studies on eye movements are conducted in the absence of postural sway, using seated participants who are subjected to head restraint (either bite bar or chin rest). ${ }^{1}$ This means that studies of eye movements generally have not considered the consequences of body sway. Similarly, studies relating vision to stance generally have not considered eye movements. Some au-

\footnotetext{
${ }^{1}$ For a critique of the study of eye movements in these "unnatural" conditions, see Steinman, Kowler, and Collewijn (1990).
} 
thors have suggested that visually controlled eye movements may be relevant to postural control (e.g., Dault et al., 2001), but very few relevant data have been collected.

Of the few studies that have examined eye movements during unrestrained stance, most are of doubtful relevance to visual guidance of eye movements, due to the inclusion of nonoptical factors such as inertial stimulation of the vestibular system (induced by voluntary head movements; Steinman \& Collewijn, 1980; or by imposed motion of the body; e.g., Welch, Bridgeman, Williams, \& Semmler, 1998) or muscle vibration (Ivanenko, Grasso, \& Lacquaniti, 1999). Skavenski, Hansen, Steinman, and Winterson (1979) studied eye movements and retinal slip during unrestrained quiet stance. They instructed participants to fixate a stationary target at optical infinity. Recordings were made of eye and head position, but there was no measurement of body motion. As noted earlier, the visual search task used by Stoffregen et al. (2000) required shifts in gaze. However, these shifts were not the focus of the study, and there was no controlled manipulation of the direction, frequency, or amplitude of gaze shifts. ${ }^{2}$

Researchers who have addressed relations between eye movements and body sway have tended to predict that sway should increase during eye movement, relative to sway when the eyes are stationary. There are two motivations for this prediction. One is based on the idea of between-task competition for limited central processing resources (Hunter \& Hoffman, 2001; Maylor \& Wing, 1996; Teasdale et al., 1993), as discussed earlier. On this view, cognitive resources devoted to the control of eye movements would not be available for the control of stance. Thus, we might expect an increase in body sway when people use eye movements to, for example, track the motion of a visible target.

The second reason that sway might increase during eye movement derives from the concept of saccadic suppression (Oblak, Gregoric, \& Gyergyek, 1985; White, Post, \& Leibowitz, 1980). A large database suggests that the pickup of information from the optic array is suppressed during saccades. If the pickup of information from the optic array were suspended during saccades, this would be similar to closing the eyes for an equivalent period. Generally, sway is greater when the eyes are closed, relative to when they are open (e.g., Edwards, 1946); hence the prediction of increased sway during visual activities that require saccades.

We know of four studies that have assessed the effects of eye movements on body sway. White et al. (1980) argued that saccadic suppression should lead to increased sway during eye movements. To test this prediction, they instructed participants to alternate gaze between two target lights that were separated by $4^{\circ}$ of horizontal visual

\footnotetext{
${ }^{2}$ Many studies have investigated relations between eye movements and locomotion to identify retinal or optical invariants used to control heading (e.g., Cutting, Alliprandini, \& Wang, 2000; Wagner, Baird, \& Barbaresi, 1981). However, posture during walking was not analyzed and it is unclear how these studies could contribute to the understanding of the relation between eye movements and stance.
} 
angle. Participants were required to stand on one leg, and were instructed to fixate whichever light was illuminated. Three conditions were relevant to this study (in the other conditions, moving visual scenes were used to simulate the optical consequences of eye movements). In the control condition, only one target light was illuminated, producing stationary fixation. In two experimental conditions, the lights were illuminated alternately at $3 \mathrm{~Hz}$ or "aperiodically every few seconds" (p. 622). The dependent variable was the ratio of the power spectra of body sway in experimental and control conditions. The hypothesis that saccadic suppression would lead to an increase in body sway when the eyes were moving was not supported; there was no difference in the power spectra of sway between the fixation and saccade conditions. Postural data were obtained using a force platform; White et al. did not measure the position or motion of the eyes or head.

Oblak et al. (1985) also hypothesized that saccadic suppression would lead to increased body sway when the eyes were moving. Standing participants fixated a stationary target, or used saccades to fixate successively target lights to the left and right of center, which were illuminated one at a time, alternating at $0.5 \mathrm{~Hz}$. Oblak et al. used electromyography to confirm that participants executed eye movements, but they did not analyze their eye movement data. Data obtained from a force platform were used to compute the mean radius of sway (the root mean square of deviations from the mean position). The hypothesis that sway would increase during eye movements was not supported. On the contrary, during eye movements there was a significant reduction in sway, relative to sway during stationary fixation. This finding is intriguing, but it cannot be taken at face value. The main reason is that Oblak et al. did not report the distance or separation of the target lights, or the amplitude of gaze shifts required to change fixation between lights; thus, we do not know whether the required gaze shifts tended to elicit head rotation (they did not measure head rotation). Head rotation is a problem for three reasons. First, it makes it more difficult to understand the role of eye movements, per se, in postural control. Second, it tends to introduce nonpostural motion of the head that complicates interpretation of postural measurements. Third, head rotation may bring into play the so-called vestibulo-ocular reflex (e.g., Steinman \& Collewijn, 1980), which could complicate interpretation of both visual and postural behavior. For these reasons, and because we were interested in relations between postural control and eye movements, per se, in this study we used motion stimuli that typically elicit eye movements without head rotation.

Kikukawa and Taguchi (1985) also found that body sway was decreased during viewing of a moving visual target (this appeared to be true for healthy participants, but not for a group of participants with peripheral vestibular disorders). The reduction in sway appeared to be concentrated in the medio-lateral (ML) sway axis. Kikukawa and Taguchi recorded motion of the center of pressure (COP) during stationary fixation, and when participants looked at two targets that were lit alternately. Here again the reduction in body sway during eye movements cannot be taken at face value, for several reasons. The amplitude of required eye movements 
$\left(20^{\circ}\right)$ was great enough to elicit movements of both the eyes and the head. In addition, the authors provided no information about the appearance of the visual stimuli. They also did not report the number or duration of trials, or inferential statistical tests on their effects.

Hunter and Hoffman (2001) measured body sway when participants fixated a stationary dot, and when they were instructed to shift gaze between several dots that appeared sequentially in different locations. Hunter and Hoffman measured the positional variability of the ML component of the COP and found that it was greater in the sequential condition than during stationary fixation. They interpreted this result in terms of "interference between the processing of visual information for the task and for the control of posture" (p. 45). However, two aspects of their methodology raise questions about the generality of their finding. First, they used heel-to-toe stance; this is not only unrepresentative of ordinary stance, but was also relatively novel for their participants, possibly requiring novel and inadequately practiced compensatory strategies. Second and perhaps more important, their eye movement condition required shifts in gaze of up to $54^{\circ}$. As noted earlier, when gaze is shifted more than $15^{\circ}$, eye movements typically are supplemented by rotation of the head (Hallett, 1986). Thus, in Hunter and Hoffman's study, it is very likely that head movements were used to fixate the targets (head movements were not measured). This means that the measured increase in $\mathrm{COP}$ variability during eye movements may have been an artifact of the use of head movements to facilitate shifts in gaze between the widely spaced targets.

This study differs from previous studies in several respects. To increase representativeness, stance was bipedal, with the feet side by side rather than heel to toe. The angular displacement of our moving targets was within the range that normally elicits shifting of gaze without rotation of the head (White et al., 1980). We did this because head rotation would tend to alter motion of the torso, which would complicate our effort to understand relations between eye movements and stance. Unlike any of the studies reviewed here, we directly measured head motion. This allowed us to determine whether head rotation was used in the presence of moving visual targets. We also conducted separate analyses of postural motion in the AP and ML axes (Hunter \& Hoffman, 2001).

\section{Summary and Predictions}

If postural control competes with suprapostural activity for limited central processing resources (e.g., Teasdale et al., 1993; Woollacott \& Shumway-Cook, 2002), then there should be degradation in postural control during eye movements that should be expressed as an increase in body sway (Hunter \& Hoffman, 2001). Alternatively, if sway is unchanged, then there should be degradation in the accuracy of eye movements (e.g., relative to eye movements made in the absence of postural control actions). By contrast, if body sway is modulated to facilitate the achievement of suprapostural tasks (Stoffregen et al., 2000; Stoffregen et al., 1999), then 
there should be a decrease in sway during visually guided eye movements (relative to stance during stationary fixation, and relative to stance during eye movements that are not visually guided), because accurate changes in the direction of gaze are easier when motion of the head and body is reduced. We assume that more precise control of the eyes is required to change the direction of gaze than to maintain stationary fixation. Previous studies have not directly contrasted these competing hypotheses and have provided ambiguous or inconsistent results. Critically, previous research has not adequately controlled for the possible influence of head rotation on gaze control or on measured body sway.

In these experiments, participants shifted their gaze between target positions, moved their (closed) eyes in time to a metronome, and held their eyes steady (during fixation of a stationary target, or with eyes closed). We predicted that (a) the amplitude and frequency of eye movements would match the amplitude and frequency of target motion (that is, we predicted that participants would successfully track the dot), (b) during visually guided eye movements, the variability of body sway would be reduced (relative to sway during fixation of stationary targets); and (c) during eye closure, posture would not be influenced by the presence or absence of eye movements. Previous research relating posture to vision has sometimes found trial effects (e.g., Pagulayan, Hayes, \& Stoffregen, 2001). For this reason, in this study we analyzed the data for possible trial effects. However, we did not make any predictions about possible trial effects.

\section{EXPERIMENT 1}

There were four conditions in Experiment 1. In the stationary target condition, participants were asked to fixate a stationary target for the duration of each trial. In the moving target condition, two dots appeared on the screen sequentially and participants were asked to shift their gaze so as to look at whichever dot was visible at any given moment. We assumed that viewing of the stationary target would require less precise control of the eyes than viewing of the moving target. On this basis, we predicted that body sway variability would be reduced in the moving target condition, relative to sway when the target was stationary. The remaining conditions permitted us to determine whether any effects of eye movements on stance were related to the visual guidance of eye movements, or to the physical movement of the eyes, per se. In the closed moving condition, participants were instructed to engage in oscillatory, lateral eye movements during eye closure. ${ }^{3}$ Sway in this condition was compared to a condition in which the eyes were closed and stationary (closed stationary). When

\footnotetext{
${ }^{3}$ Oblak et al. (1985) included a condition where eye movements were made with eyes closed, but did not report any basis for pacing of frequency of these movements. Electromyography confirmed that participants moved their eyes as instructed. However, Oblak et al. did not report inferential statistics involving sway during eye movements with the eyes closed.
} 
the eyes are closed, eye movements are not visually guided, that is, eye movements are not organized or evaluated with respect to targets in the environment, and so small variations in body sway should not influence such eye movements. A finding that body sway was influenced by eye movements during eye closure could undermine our assertion that the relation between sway and eye movements is functional. There was not an obvious reduction in cognitive demand in the eyes-closed condition. This is because we required participants to move their eyes in time with a metronome, which constrained the timing (but not the direction or amplitude) of eye movements. The metronome also had the benefit of ensuring that the frequency of eye movements was the same in eyes-open and eyes-closed conditions.

Separate measurement of head and torso position permitted us to evaluate several hypotheses. First, we could determine whether eye movements influenced only torso motion or motion of both the torso and head. Second, the collection of data on head position allowed us to determine whether participants rotated their heads. Given the small amplitude of gaze change needed, this was unlikely; however, it was important to evaluate the possibility.

\section{Method}

Participants. Fourteen undergraduate students ( 8 men, 6 women) from the University of Cincinnati participated in exchange for course credit. Participants reported no history of disease or malfunction of the vestibular apparatus, or of postural instability, recurrent dizziness, or falls. Due to apparatus constraints, participant height was limited to a maximum of $183 \mathrm{~cm}$. Height ranged between $157.5 \mathrm{~cm}$ and $183 \mathrm{~cm}(M=170 \mathrm{~cm})$. Age ranged from 18 to 29 years $(M=20$ years $)$. Three participants had corrected vision (glasses or contacts). Participants were treated in accordance with the Ethical Principles of Psychologists and Code of Conduct (American Psychological Association, 1992).

Apparatus. Postural sway was recorded using a 6-df (degrees of freedom) magnetic tracking system (Flock of Birds, Ascension Technologies, Inc., Burlington, Vermont). One receiver was attached to a bicycle helmet worn by the participant, while a second receiver was attached to the skin between the shoulders (approximately at the seventh cervical vertebra) using cloth medical tape. Each receiver was sampled at $25 \mathrm{~Hz}$, and the data were stored on a computer for later analysis. Visual stimuli were generated using PsyScope (Cohen, MacWhinney, Flatt, \& Provost, 1993), and presented on a Macintosh G3 computer with a $43 \mathrm{~cm}$ Apple Studio Display.

Procedure. The experiment was conducted at the University of Cincinnati. The visual target consisted of a filled red circle on a white background. Luminance of the background was approximately $108.44 \mathrm{~cd} / \mathrm{m}^{2}$, and luminance of the target was approximately $26.07 \mathrm{~cd} / \mathrm{m}^{2}$. The contrast ratio was approximately $1: 4$. Participants stood with their eyes $100 \mathrm{~cm}$ from the display. At this distance, the target cir- 
cles subtended approximately $1.15^{\circ}$ of visual angle. In the stationary target condition, the visual target appeared in the center of the display and remained there for the duration of the trial. In the moving target condition, the target was presented in two positions, alternating between positions at $0.583 \mathrm{~Hz}$ to produce apparent motion. The target first appeared $9.75 \mathrm{~cm}$ to the left of the center of the display for $1 \mathrm{sec}$, at which point it disappeared, reappearing approximately $9.75 \mathrm{~cm}$ to the right of the center of the display, where it was again visible for $1 \mathrm{sec}$ (maximum displacement approximately $19.5 \mathrm{~cm}$, subtending $11^{\circ}$ of visual angle in the horizontal plane). The $11^{\circ}$ gaze shifts required in the moving target condition were well within the range that typically is accomplished with eye movements alone, that is, without supporting head movements (Hallet, 1986). Participants were shown an example of the moving target condition to ensure they fully understood the task.

In the third condition (closed moving), participants were instructed to move their eyes while they were closed. They were asked to move their eyes with the same amplitude as in the moving target condition (they were allowed to practice eyes-closed eye movements after viewing the stimulus for the moving target condition). Eye movements were paced to the sound of a metronome, which emitted a tone every $1.715 \mathrm{sec}$. Participants were instructed to move their eyes once at each tone, so that a full cycle (i.e., two movements) occurred at a frequency of $0.583 \mathrm{~Hz}$. We sought to reproduce the $0.5 \mathrm{~Hz}$ used in previous studies, but 0.583 was the lowest frequency that our metronome could produce. This fact dictated the frequency of tones in the closed moving condition and, therefore, in the moving target condition. In the fourth condition (closed stationary), participants were instructed to close their eyes but keep them pointing straight ahead.

Each participant performed 12 trials (three in each condition), each of which lasted $65 \mathrm{sec}$. Condition order was counterbalanced across participants. Participants donned the bicycle helmet with the receiver attached to the back of it. An experimenter attached the second receiver. Participants were asked to stand approximately $100 \mathrm{~cm}$ from the display, with their feet together. The display was adjusted so that the top of the screen was approximately level with the participant's eyeheight. In all trials, participants were given the option of letting their arms hang at their sides, putting their hands in their pockets, or holding their hands clasped in front of or behind them. Whichever position they chose, they were asked to keep the same position for each trial, and not to move their hands during trials.

In conformity with University of Cincinnati institutional review board requirements, participants were required to sit and take a break after the first six experimental trials. This was done to minimize the possibility of fatigue or other adverse side effects of prolonged stance (Smart, Pagulayan, \& Stoffregen, 1998).

\section{Results}

The dependent variables were the positional variability of the head and torso in fore-aft and lateral motion. These were operationalized as the standard deviation 
of the head and torso displacements, analyzed separately for the AP and ML axes. The independent variable was condition (stationary target, moving target, closed stationary, closed moving). Separate analyses of variance (ANOVAs) were conducted on AP and ML motion of the head, and on AP and ML motion of the torso. Summary data (collapsed across trials) are presented in Figure 1. Data from representative trials are presented in Figure 2.

Torso ML. The main effect of condition was significant, $F(3,39)=22.39$, $p$ $<.05$, partial $\eta^{2}=0.63 .{ }^{4}$ The main effect of trial was not significant, $F(2,26)=$ $1.36, p>.05$, nor was the Condition $\times$ Trial interaction, $F(6,78)<1$, ns. Planned $t$ tests (using a criterion alpha of .0167) ${ }^{5}$ revealed that there was less variability in the moving target condition $(\mathrm{M}=0.216 \mathrm{~cm})$ than in the stationary target condition $(M=0.294 \mathrm{~cm}) ; t(13)=4.80, p<.0167$, as predicted. There was also less variability in the moving target condition than in the closed moving condition ( $\mathrm{M}$ $=0.385 \mathrm{~cm}) ; t(13)=6.68, p<.0167$, as predicted. There was less variability in the stationary target condition than in the closed stationary condition $(M=0.437$ $\mathrm{cm}) ; t(13)=3.64, p<.0167$, replicating classical effects (e.g., Edwards, 1946). Finally, variability in the closed stationary condition did not differ from that in the closed moving condition, $t(13)=2.05, p>.0167$. Thus, the influence of eye movements on torso motion occurred only when the eyes were open.

Torso AP. The main effect of conditions was significant, $F(3,39)=34.07$, $p$ $<.05$, partial $\eta^{2}=0.72$. The main effect of trial was not significant, $F(2,26)=$ $2.49, p>.05$, but the Trial $\times$ Condition interaction was significant, $F(6,78)=$ $2.39, p<.05$, partial $\eta^{2}=0.16$. The Trial $\times$ Condition interaction is illustrated in Figure 3 . The interaction seems to indicate that when the eyes were moving, postural variability followed an inverted $\mathrm{U}$, being greater in Trial 2 than in Trials 1 and 3. When gaze was stationary, postural variability decreased over trials (when the eyes were open), or was constant over trials (when the eyes were closed). We did not predict this interaction, and we do not have a clear interpretation of it.

Planned comparisons on the main effect of conditions revealed that the moving target condition $(\mathrm{M}=0.234 \mathrm{~cm})$ did not differ from the stationary target condition, $M=0.237 \mathrm{~cm}, t(13)=0.165, p>.0167$. The moving target condition was significantly less variable than the closed moving condition, $M=0.337 \mathrm{~cm}, t(13)$ $=6.51, p<.0167$, as predicted. The stationary target condition was significantly less variable than the closed stationary condition, $M=0.363 \mathrm{~cm}, t(13)=6.74, p<$ .0167, replicating classical effects (e.g., Edwards, 1946; Riley et al., 1997). The closed stationary condition did not differ from the closed moving condition, $t(13)$ $=2.28, p>.0167$.

\footnotetext{
${ }^{4}$ The effect size was computing using SPSS and reporting the partial $\eta^{2}$ statistic.

${ }^{5}$ The criterion alpha was selected using the Bonferroni adjustment, taking into account that we conducted some statistical comparisons between Experiments 1 and 2 (see later).
} 


\section{Torso}
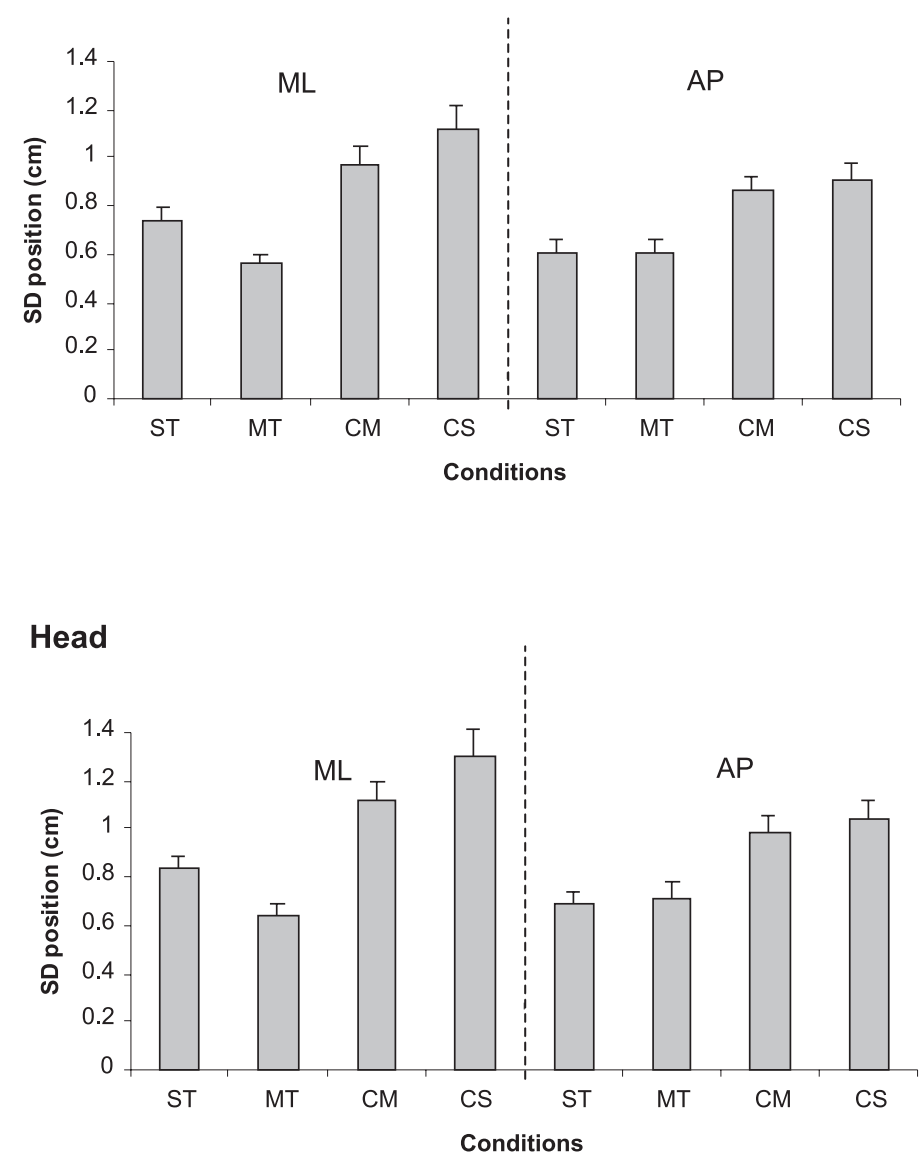

FIGURE 1 Means of the positional variability of the head and torso in the AP and ML axes, Experiment 1 . ST $=$ stationary visual target; $\mathrm{MT}=$ apparent motion of the visual target at $0.583 \mathrm{~Hz} ; \mathrm{CM}=$ eyes closed with instructions to move the eyes in time with the metronome at $0.583 \mathrm{~Hz} ; \mathrm{CS}=$ eyes closed with instructions to hold the eyes stationary. Error bars represent the standard error of the mean.

Head ML. The main effect of condition was significant, $F(3,39)=22.34, p$ $<.05$, partial $\eta^{2}=0.63$. The main effect of trial was not significant, $F(2,26)=$ $1.64, p>.05)$; nor was the Condition $\times$ Trial interaction, $F(6,78)<1$, ns.

Planned $t$ tests revealed that, as predicted, variability of head motion during the moving target condition, $M=0.252 \mathrm{~cm}$, was significantly less than during the stationary target condition, $M=0.325 \mathrm{~cm}, t(13)=3.86, p<.0167$. As predicted, variability of head motion during the moving target condition was significantly less 
Open stationary

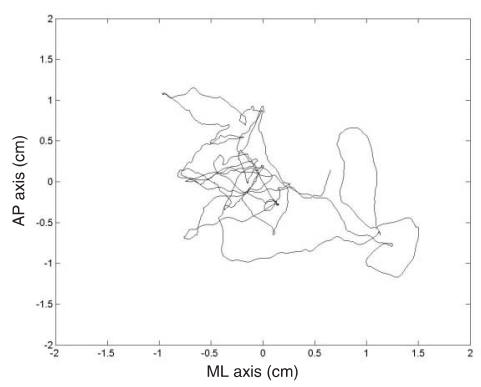

Closed stationary

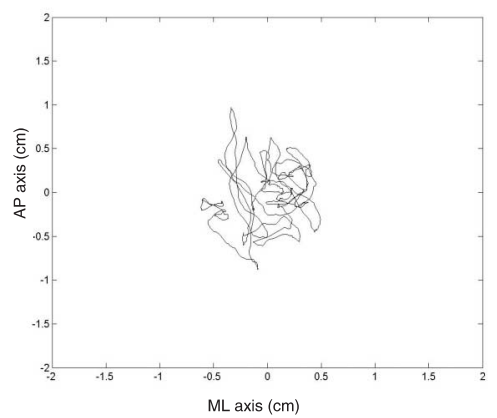

Open moving

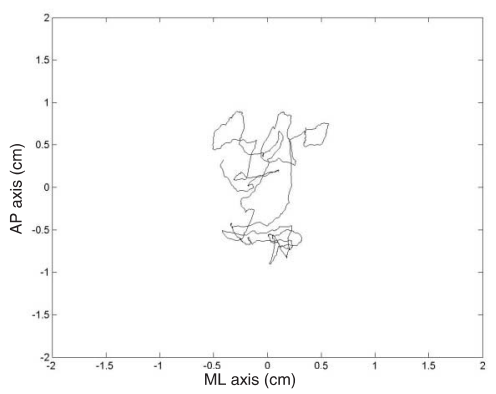

Closed moving Participant 12

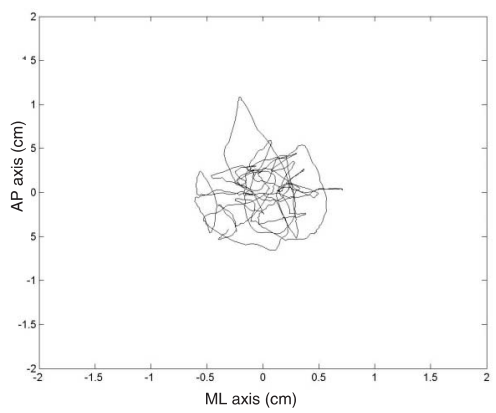

FIGURE 2 Representative head/torso position data for one participant in Experiment 1.

\section{Torso, AP axis}

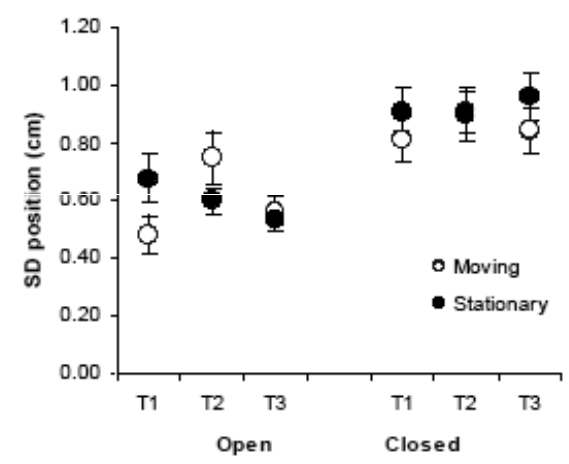

FIGURE 3 The significant Trial $\times$ Condition interaction, Experiment 1 . 
than during the closed moving condition, $M=0.435 \mathrm{~cm}, t(13)=7.08, p<.0167$. In addition, variability of head motion during the stationary target condition was significantly less than during the closed stationary condition, $M=0.511 \mathrm{~cm}, t(13)$ $=3.82, p<.0167$, replicating classical effects (e.g., Edwards, 1946). Finally, ML head motion during the closed moving condition was not significantly different than during the closed stationary condition, $t(13)=2.26, p>.0167$, confirming our prediction.

Head AP. The main effect of condition was significant, $F(3,39)=30.38, p<$ .05 , partial $\eta^{2}=0.70$. The main effect of trial was significant, $F(2,26)=4.07, p<$ .05 , partial $\eta^{2}=0.24$, but the Trial $\times$ Condition interaction was not significant, $F(6,78)=2.09, p>.05$. Means and standard deviations for the trials were as follows: Trial $1, M=0.81 \mathrm{~cm}, S D=0.36 \mathrm{~cm}$; Trial $2, M=0.92 \mathrm{~cm}, S D=0.37 \mathrm{~cm}$; and Trial $3, M=0.83 \mathrm{~cm}, S D=0.33 \mathrm{~cm}$.

Planned comparisons on the main effect of conditions revealed that AP motion of the head did not differ during the moving target and stationary target conditions, $M=0.281$ and $0.266 \mathrm{~cm}$, respectively; $t(13)=0.575, p>.0167$. AP head motion during the moving target condition was significantly reduced relative to the closed moving condition, $M=0.389 \mathrm{~cm}, t(13)=5.43, p<.0167$. During the stationary target condition, AP head motion was significantly less than during the closed stationary condition, $M=0.412 \mathrm{~cm}, t(13)=7.83, p<.0167$, replicating classical effects (e.g., Edwards, 1946). During the closed stationary condition, AP head motion did not differ from the closed moving condition, $t(13)=1.95, p>$ .0167. Finally, post hoc $t$ tests on the main effect of trial revealed that AP motion of the head was greater during Trial 2 than during either Trial $1, t(55)=1.99, p<.05$, or Trial $3, t(55)=2.18, p<.05$. Trials 1 and 3 did not differ, $t(55)<1$, ns.

Head rotation. We computed head rotation about the vertical axis. This allowed us to evaluate the possibility that participants used head movements to maintain gaze on the moving target, rather than (or in addition to) eye movements. Means and standard deviations for the conditions were as follows: stationary target, $M=1.08^{\circ}, S D=1.08^{\circ}$; moving target, $M=1.31^{\circ}, S D=1.22^{\circ}$; closed moving, $M=0.48^{\circ}, S D=0.85^{\circ}$; closed stationary, $M=1.24^{\circ}, S D=0.45^{\circ}$. An ANOVA revealed that there was not a significant effect of condition on head rotation around the vertical axis, $F(3,123)<1$, ns.

\section{Discussion}

For both the head and torso, sway in the ML axis was reduced when the target moved, relative to when it was stationary. Sway in the closed moving condition was less variable than in the closed stationary condition, but this was true only for head ML. That is, there was an effect on the head, but not on the torso. Variability of head motion in the moving target condition was less than in the closed moving 
condition, for both head and torso, in both axes. Finally, there was no difference among conditions in the rotation of the head around its vertical axis.

\section{Postural Stabilization of Visually Guided Eye Movements}

The results from Experiment 1 confirmed most of our predictions. Postural sway variability in the ML axis was reduced when looking at a moving target, relative to sway when looking at a stationary target. Experiment 1 provides support for the claim that the control of posture can be influenced by suprapostural visual tasks. The purpose of this experiment was to investigate relations between eye movements and body sway under conditions in which other factors, such as target distance and the cognitive content of tasks were held constant. Together with the findings of Oblak et al. (1985), Experiment 1 makes it clear that visually guided eye movements alone are sufficient to influence the organization of body sway.

\section{Head Rotation}

There was no effect of condition on rotation of the head around its vertical axis. That is, participants did not use head rotation differentially across conditions. This means that the most likely cause of the postural effects of target motion is eye movement per se. It may be important that the lack of head rotation effects corresponded with the presence of head and torso effects in the ML axis, indicating that the level of fine-tuning of postural changes with respect to the suprapostural task. These results suggest the possibility that body sway (in the ML axis) might be used to stabilize eye movements that are less than $11^{\circ}$ in amplitude, with head rotation (perhaps augmented by sway) being used when eye movements exceed $11^{\circ}$.

\section{Eye Movements When the Eyes Were Closed: Torso Motion}

The main purpose of asking participants to move their eyes while they were closed was to determine whether postural control would be influenced by eye movements that were not visually guided. In assessing this possibility, the central comparison was between torso motion with the eyes closed and stationary, and torso motion with the eyes closed and moving. When the eyes were closed, there was not a significant difference in the variability of torso motion in the eyes-stationary and eyes-moving conditions in either the AP or ML axes. This contrasts with the influence of eye movements on torso motion when the eyes were open: Visually guided eye movements produced a reduction in torso motion, but eye movements that did not rely on visual guidance had no effect on torso motion. This contrast supports our argument that the relation between postural sway and eye movements is func- 
tional, that is, that posture is used to facilitate the control of eye movements that are visually guided.

The variability of torso position was reduced when eye movements were visually guided (the moving target condition), relative to when they were not (the closed moving condition). This confirms that the differences in torso motion between the moving target and stationary target conditions were related to the control of the visual system relative to the visible environment (i.e., to visual performance), and not to the presence of eye movement, per se.

\section{Eye Movements When the Eyes Were Closed: Head Motion}

When the eyes were closed eye movements did not influence motion of the torso, but they did influence motion of the head. In the ML axis there was a significant decrease in head motion during the closed moving condition, relative to sway in the closed stationary condition. This effect confirms that participants actually modified their behavior in response to our instruction to move the eyes while they were closed.

The fact that there was an effect on ML head motion of eye movements when the eyes were closed was contrary to our prediction. However, the direction of the effect was contrary to predictions that could be motivated by the idea that postural and ocular control compete for a limited pool of central processing resources. The resource competition view can predict reduction in performance of one or both of two simultaneous tasks and, if the two tasks do not exhaust the available resources, it can predict that performance of the combined tasks will be equivalent to the performance of each task alone. But the resource competition view does not appear to offer any basis for predicting that performance of either task will improve when they are combined.

The reduction in variability of ML head motion during eyes-closed eye movements may suggest some relation between head motion and the mechanics of the oculomotor system. However, other interpretations are possible. One interpretation arises from the fact that in the closed moving condition eye movements were paced to the sound of a metronome. In this condition, participants were obliged to pay attention to the sound of the metronome, independent of their need to move the eyes. Listening to the metronome may have constituted a suprapostural task. It is possible that the reduced variability of ML head motion facilitated the task of listening to the metronome; such an effect would be functional. That is, participants may have engaged in postural stabilization of listening. Additional research will be required to determine whether changes in ML head motion are related to listening, to the mechanics of nonvisual eye movements, or to some other cause.

Another interpretation of the condition effect on ML head motion in the absence of an effect on torso motion relates to the information needed to control eye movements. To produce eye movement in phase with the metronome, participants 
needed information about how their eyes were moving. With the eyes closed, such information was not available visually, and so it seems likely that the required information would have been obtained proprioceptively, for example, in feedback from ocular muscles moving the eyes in the medio-lateral direction. The pickup of such information might be complicated by simultaneous motion of the head in the same direction: Proprioceptive information about head movement would be irrelevant to the eye movement task. For this reason, a reduction in head motion (especially in the ML axis) may have made it easier for participants to detect the proprioceptive information needed to execute the novel task of moving their eyes laterally during eye closure. It may be that participants reduced head movement to better perceive (and control) the motion of their eyes with respect to the metronome, similar to the reduction in head rotation that has been observed during various locomotor tasks (e.g., Berthoz, 1990).

\section{EXPERIMENT 2}

In Experiment 2, we measured eye movements, using electro-oculography (EOG). EOG is the only technology that permits measurement of movements when the eyes are closed as well as when they are open. Our main purpose in Experiment 2 was to confirm that participants moved their eyes when instructed to do so and to determine the amplitude of eye movements. The conditions and manipulations were the same as in Experiment 1 and, accordingly, we made the same predictions about relations between body sway and visually guided eye movements.

\section{Method}

Participants. Fourteen undergraduates from the University of Minnesota participated as volunteers; none had participated in Experiment 1. Participants reported no history of disease or malfunction of the vestibular apparatus, or of postural instability, recurrent dizziness, or falls. We did not restrict participation on the basis of height. Rather, the height of the display monitor was adjusted individually for participants. Participants' height ranged between $152.4 \mathrm{~cm}$ and $195 \mathrm{~cm}(\mathrm{M}=$ $173.1 \mathrm{~cm})$. Age ranged from 19 to 25 years $(M=21$ years). Three participants had corrected vision (glasses or contacts).

Apparatus. Displays were created using PsyScope, as in Experiment 1, and were presented on a color flat screen monitor measuring $50.8 \mathrm{~cm}$ diagonally. Eye position and movement were measured using a standard EOG system (Biopac Systems, Inc., Goleta, California). The EOG system was positioned immediately to the participant's right. This was done because the cables connecting the electrodes to the EOG electronics unit were $114 \mathrm{~cm}$ long (this fact is important to our interpretation of some of the results). The EOG electronics unit was connected to a PC 
that sat on a desk on the participant's left. The cable connecting the electronic unit to the computer passed in front of the participant. Eye position was sampled at 62.5 $\mathrm{Hz}$. Postural data (head and torso motion) were measured using the Flock of Birds, with each receiver sampled at $47 \mathrm{~Hz}$.

Procedure. The experiment was conducted at the University of Minnesota. The conditions and procedure were the same as in Experiment 1, except for the use of the EOG system. Participants were asked to stand with their toes on a line on the floor, so that their eyes were approximately $100 \mathrm{~cm}$ from the center of the monitor. The EOG system was calibrated before each trial, using the method recommended by the manufacturer.

In conformity with University of Minnesota institutional review board requirements, participants were required to sit and take a break after the first six experimental trials. This was done to minimize the possibility of fatigue or other adverse side effects of prolonged stance (Smart et al., 1998).

\section{Results}

Postural motion. The data are summarized in Figures 4 and 5. We conducted separate repeated measure ANOVAs on data for the AP and ML axes, and for the head and torso. For each significant effect in the ANOVAs, planned $t$ tests were conducted. These $t$ tests were not independent, and for this reason, we applied the Bonferroni inequality to adjust the criterion alpha. For each ANOVA, four $t$ tests were planned, yielding a criterion alpha for each test of 0.0167 , as explained earlier.

Torso ML. The main effect of condition was significant, $F(3,39)=4.27, p<$ .05 , partial $\eta^{2}=0.25$. The main effect of trial was significant (Figure 5), $F(2,26)=$ $8.20, p<.05)$, partial $\eta^{2}=0.39$; but the Condition $\times$ Trial interaction was not significant, $F(6,78)<1$, ns. We did not predict significant main effects of trial on postural motion in Experiment 2. The meaning of these effects is considered in the Discussion section.

Planned $t$ tests revealed that there was less variability in the moving target condition, $M=0.122 \mathrm{~cm}$, than in the stationary target condition, $M=0.160 \mathrm{~cm}, t(13)$ $=3.489, p<.0167$, as predicted. The moving target and closed moving conditions did not differ (closed moving $M=0.138 \mathrm{~cm}) ; t(13)=1.166, p>.0167$. The stationary target and closed stationary conditions also did not differ (closed stationary $M=0.150 \mathrm{~cm}$ ), $t(13)=.897, p>.0167$, not replicating classical effects (e.g., Edwards, 1946; Riley et al., 1997). Finally, variability in the closed stationary condition did not differ from that in the closed moving condition, $t(13)=1.254, p>$ .0167 . Thus, the influence of eye movements on torso motion occurred only when the eyes were open. 


\section{Torso}

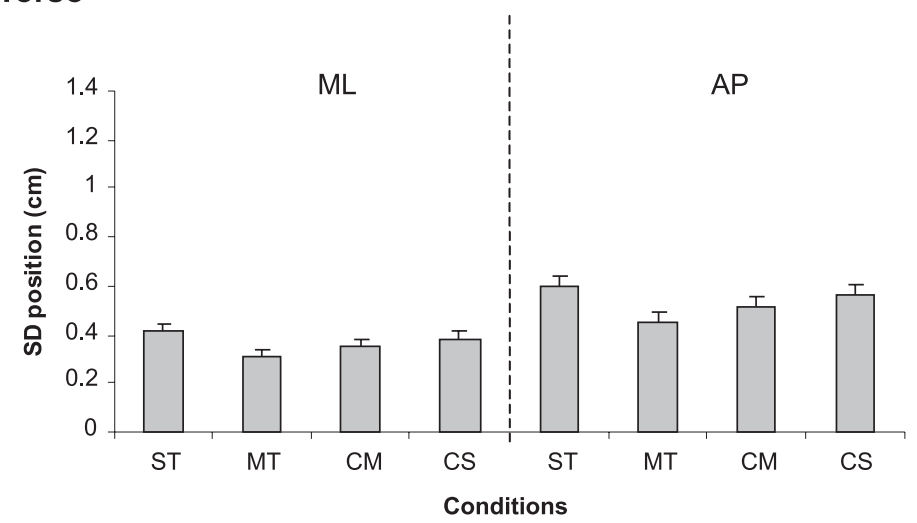

\section{Head}

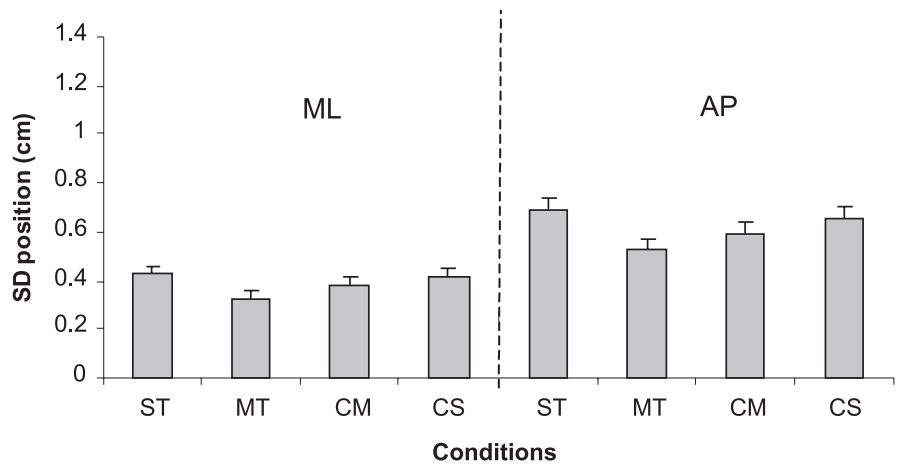

FIGURE 4 Means of the positional variability of the head and torso in the AP and ML axes, Experiment 2. ST $=$ stationary visual target; $\mathrm{MT}=$ apparent motion of the visual target at $0.583 \mathrm{~Hz} ; \mathrm{CM}=$ eyes closed with instructions to move the eyes in time with the metronome at $0.583 \mathrm{~Hz} ; \mathrm{CS}=$ eyes closed with instructions to hold the eyes stationary. Error bars represent the standard error of the mean.

Torso AP. The main effect of condition was significant, $F(3,39)=4.33, p<$ .05 , partial $\eta^{2}=0.25$. The main effect of trial was significant (Figure 5), $F(2,26)=$ $7.26, p<.05$, partial $\eta^{2}=0.36$, but the Condition $\times$ Trial interaction was not significant, $F(6,78)<1$, ns.

Planned comparisons on the main effect of condition revealed that motion in the moving target condition, $M=0.178$, differed from the stationary target condition, $M=0.238 \mathrm{~cm}, t(13)=3.526, p<.0167$. Sway in the moving target condi- 


\section{Torso}
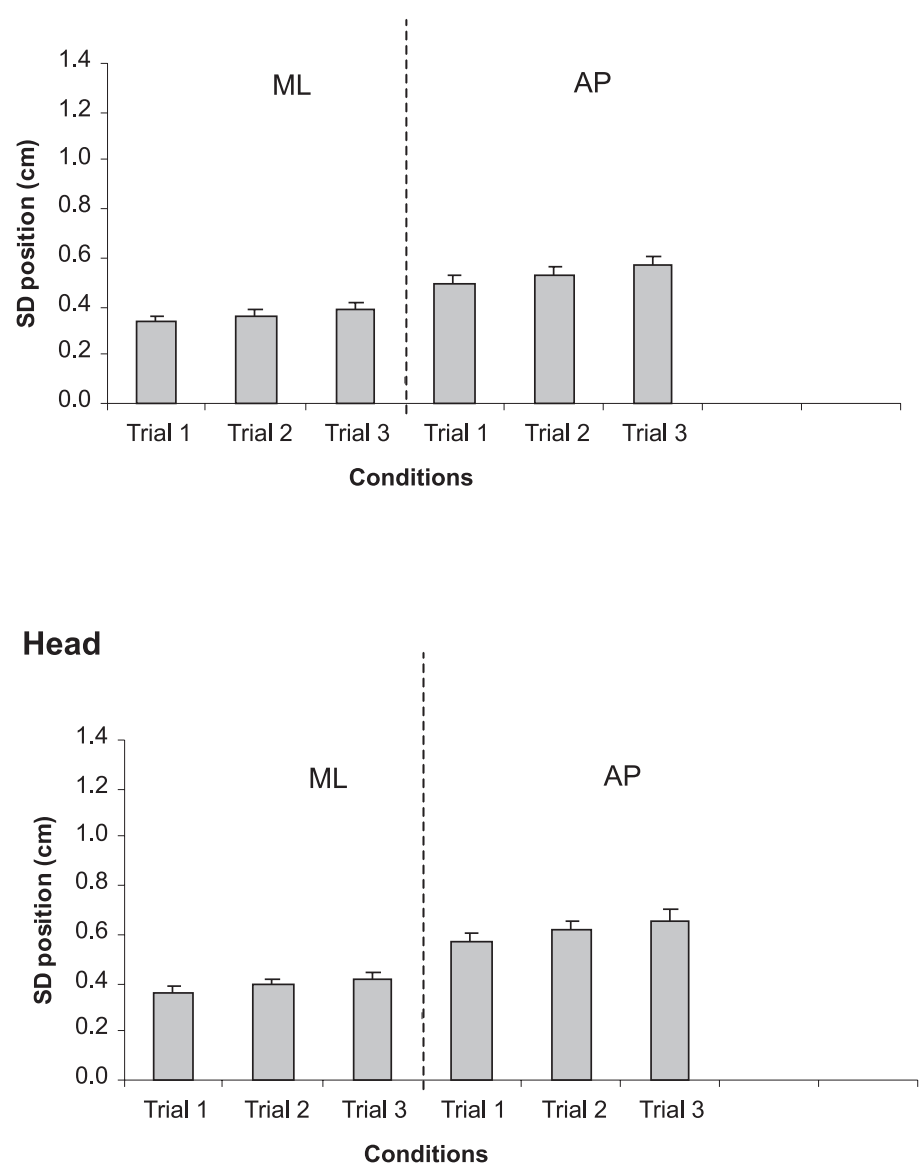

FIGURE 5 The significant main effects of trials, Experiment 2. A: Torso. B: Head. T1, T2, T3: Trials 1, 2, and 3, respectively. Error bars represent the standard error of the mean.

tion did not differ from the closed moving condition, $M=0.201 \mathrm{~cm}, t(13)=1.142$, $p>.0167$. Similarly, sway did not differ in the stationary target and closed stationary conditions, not replicating classical effects, $t(13)=1.083, p>.0167$ (closed stationary $M=0.219 \mathrm{~cm}$ ). Finally, as predicted, sway in the closed stationary condition did not differ from the closed moving condition $t(13)=1.171, p>.0167$.

Head ML. The main effect of condition was significant, $F(3,39)=3.61, p<$ .05 , partial $\eta^{2}=0.22$. The main effect of trial was significant (Figure 5), $F(2,26)=$ $5.55, p<.05$, partial $\eta^{2}=0.30$; but the Condition $\times$ Trial interaction was not significant, $F(6,78)<1$, ns. 
Planned $t$ tests revealed that, as predicted, variability of head motion during the moving target condition, $M=0.131 \mathrm{~cm}$, was significantly less than during the stationary target condition, $M=0.170 \mathrm{~cm}, t(13)=3.552, p<.0167$. As previously, variability of head motion during the moving target condition did not differ compared to variability of head motion during the closed moving condition, $M=0.149$ $\mathrm{cm}, t(13)=1.289, p>.0167$. In addition, variability of head motion during the stationary target condition was not significantly different from that of the closed stationary condition, $M=0.161 \mathrm{~cm}, t(13)=.756, p>.0167$, again not replicating classical effects. Finally, ML head motion during the closed moving condition did not differ from that in the closed stationary condition, $t(13)=1.002, p>.0167$.

Head AP. The main effect of condition was significant, $F(3,39)=3.70, p<$ .05 , partial $\eta^{2}=0.22$. The main effect of trial was significant (Figure 5$), F(2,26)=$ $5.23, p<.05$, partial $\eta^{2}=0.29$, but the Trial $\times$ Condition interaction was not significant, $F(6,78)<1$, ns.

Planned comparisons on the main effect of condition revealed that AP motion of the head differed in the moving target and stationary target conditions, $M=0.207$ and 0.271 , respectively; $t(13)=3.524, p<.0167$. However, in the moving target and closed moving conditions, $M=0.233 \mathrm{~cm}$, AP head motion did not differ, $t(13)=$ $-1.212, p>.0167$. Similarly, in the stationary target and closed stationary conditions, $\mathrm{M}=0.255 \mathrm{~cm}$, AP head motion did not differ, $t(13)=.729, p>.0167$, nor did the closed stationary and closed moving conditions, $t(13)=1.123, p>.0167$.

Comparison of Experiments 1 and 2. Our purposes in Experiment 2 were to show that participants moved their eyes when they were instructed to do so, that eye movements were similar in amplitude and frequency to the apparent motion of the visual targets, and to confirm that these effects corresponded to reductions in the variability of postural sway when participants were shifting their gaze (i.e., in the moving target condition), relative to sway when gaze was stationary (the stationary target condition) or when the eyes were closed (the closed stationary and closed moving conditions). With respect to each of these goals, Experiment 2 was successful.

In Experiment 2, sway did not differ when the eyes were closed (the closed stationary condition) relative to when they were open (the stationary target condition). This deviates from Experiment 1, and from classical findings that sway tends to increase when the eyes are closed. The absence of this effect in Experiment 2 is not directly relevant to the purposes of the experiment, as described here. However, the failure to replicate this classical effect is puzzling. In an attempt to understand this unusual result, we compared the data from the two experiments, in the same conditions. For each condition we conducted independent $t$ tests for each body segment (head and torso) in each axis (AP and ML). In the closed stationary condition, each of the four $t$ tests was significant: head AP, $t(13)=-5.07, p<$ .0167 ; head ML, $t(13)=-7.512, p<.0167$; torso AP, $t(13)=-5.131, p<.0167$; 
and torso ML, $t(13)=-6.917, p<.0167$. In each case, sway in Experiment 2 was less than in Experiment 1 (compare Figures 1 and 4). This was true also in the closed moving condition: head AP, $t(13)=-5.279, p<.0167$; head ML, $t(13)=$ $-8.01, p<.0167$; torso AP, $t(13)=-5.084, p<.0167$; torso ML, $t(13)=-6.832, p$ $<.0167$. These results indicate that in Experiment 2, sway with the eyes closed was reduced relative to typical situations (and relative to sway in Experiment 1).

Similar results were obtained in the eyes-open conditions. For the stationary target condition, there were significant reductions in sway in Experiment 2, relative to the same condition in Experiment 1, but only in the ML axis: head AP, $t(13)$ $=-1.907, p>.0167$; head ML, $t(13)=-4.017, p<.0167$; torso AP, $t(13)=$ $-2.124, p>.0167$; torso ML, $t(13)=-3.583, p<.0167$. For the moving target condition, sway in Experiment 2 was reduced relative to Experiment 1, for both the head and torso, but again only in the ML axis: head AP, $t(13)=-1.852, p>.0167$; head ML, $t(13)=-4.866, p<.0167$; torso AP, $t(13)=-1.719, p>.0167$; torso $\mathrm{ML}, t(13)=-4.763, p<.0167$. Overall, our post hoc tests revealed that sway in Experiment 2 was reduced relative to sway in Experiment 1. We offer an interpretation of this effect in the Discussion section.

Head rotation. We computed head rotation about the vertical axis. This allowed us to evaluate the possibility that participants used head movements, rather than (or in addition to) eye movements, to maintain gaze on the moving target. Means and standard deviations for the conditions were as follows: stationary target, $M=0.60^{\circ}, S D=0.21^{\circ}$; moving target, $M=0.63^{\circ}, S D=0.29^{\circ}$; closed moving, $\mathrm{M}=0.59^{\circ}, \mathrm{SD}=0.20^{\circ}$; closed stationary $\mathrm{M}=0.73^{\circ}, \mathrm{SD}=0.39^{\circ}$. An ANOVA revealed that there was not a significant effect of condition on head rotation around the vertical axis, $F(3,123)=1.04, p>.05$.

Eye movements. Due to technical problems, eye movement data were dropped from three trials in the closed stationary condition, and from seven trials in the stationary target condition. In the stationary target and closed stationary conditions participants were instructed not to move their eyes. For these conditions, we calculated the standard deviation of horizontal eye position. For the stationary target condition, the mean standard deviation of eye position was $4.2^{\circ}$, whereas for the closed stationary condition it was $7.0^{\circ}$. In the moving target and closed moving conditions participants were asked to move their eyes. For these conditions, we first computed the mean eye position during each fixation (i.e., the period between successive eye movements), separately for fixation on the right and left sides. The difference in position between the mean left and right positions was considered to be the amplitude of each individual eye movement. Using these data, we calculated the mean and standard deviation of eye movement amplitudes for each trial. In the moving target condition, the mean amplitude of eye movements was $11.2^{\circ}$, and the standard deviation of this mean was $1.5^{\circ}$. In the closed moving 
condition, the mean amplitude of eye movements was $73.6^{\circ}$, and the standard deviation of this mean was $9.0^{\circ}$.

We analyzed the frequency of eye movements. We did this by counting the total number of eye movements in each trial, and dividing by the duration of the trial. Across trials and participants, the mean and standard deviation of eye movement frequency were as follows: moving target condition, $M=0.54 \mathrm{~Hz}, S D=0.02 \mathrm{~Hz}$; closed movement condition, $\mathrm{M}=0.54 \mathrm{~Hz}, \mathrm{SD}=0.02 \mathrm{~Hz}$. On the basis of these data, we concluded that participants moved their eyes when requested to do so, and that in the moving target condition they moved them at the same amplitude and frequency as the visual targets.

\section{Discussion}

The eye movement data were supportive of our hypotheses. Participants moved their eyes when requested, and held them still when requested. Eye movements closely matched the frequency of stimulus motion, both with visual target motion (when the eyes were open), and with metronome pace (when they were closed). When the eyes were open, the angular amplitude of eye movements closely matched the angular amplitude of stimulus motion. When the eyes were closed, the angular amplitude of eye movements was greater than the instructed amplitude; this result is consistent with findings from previous studies (e.g., Kikukawa \& Taguchi, 1985; Oblak et al., 1985), and suggests that a person's knowledge of eye movement amplitude is strongly dependent on visible referents, rather than being related to nonvisual referents (e.g., internal coordinate systems).

As in Experiment 1, head rotation was not used with moving visible targets. In addition, head rotation was not used when participants were instructed to move their eyes in time with the metronome. This is especially interesting given that eye movements were very large when the eyes were closed, $M=73.59^{\circ}$, as opposed to those in the open movement condition, $\mathrm{M}=11.16^{\circ}$. Thus, we can conclude with some confidence that the differences in sway between moving target and stationary target conditions were not caused by differential rotation of the head around the vertical axis (i.e., in the plane of target motion).

With respect to the hypotheses that motivated our study, the postural data generally replicated the findings of Experiment 1 . In some cases, the results of Experiment 2 can be seen as having been stronger than in Experiment 1. In Experiment 1, reductions in sway in the moving target condition were limited to head and torso motion in the ML axis. By contrast, in Experiment 2 such reductions were observed for both the ML and AP axes, for both the head and torso. In other cases, effects in Experiment 2 can be seen as having been weaker than in Experiment 1. In Experiment 1 , sway was reduced during visually guided eye movements (the moving target condition) relative to sway during eye movements that were not visually guided (the closed moving condition) for both the head and torso in both the ML and AP axes. By contrast, in Experiment 2 no such reductions were observed. 
One difference not related to our hypotheses was that overall sway did not increase when the eyes were closed. This most likely reflects caution on the part of participants, related to the proximity of the EOG apparatus (electronics unit) and/or the cable connecting the EOG unit to the PC. That is, we believe that participants reduced their overall sway so as to ensure that they did not bump into the experimental apparatus. A similar effect was reported by Stoffregen et al. (1999), who compared sway in a large room with sway when participants stood in a small booth $(1 \mathrm{~m}$ by $0.8 \mathrm{~m})$. In three experiments, they found that sway variability was reduced during stance in the booth relative to stance when the booth was absent. By varying the transparency of the booth walls, they manipulated the motion parallax and optical velocities created by body sway. Their results showed that the reduction in sway during stance in the booth was related to the physical size of the booth rather than to parameters of sway-generated optic flow, and they suggested that the reduction in sway might have arisen from participants' desire to avoid bumping into the walls.

A second notable difference was that in Experiment 2 the main effect of trial was significant for each of our dependent variables. These trial effects accounted for large portions of the variance; in some cases, larger than the portion accounted for by the condition effects. In each of the dependent variables, postural motion increased over trials. We did not predict the trial effects in either experiment. Trial effects on postural sway in the context of suprapostural tasks have been reported by Pagulayan et al. (2001), who also did not predict them. These trial effects may be related to an effect observed by Stoffregen et al. (2000), who asked participants to shift their attention from a nearby target to a more distant target (or vice versa) in the middle of a trial. They found that sway changed during trials as a function of fixation distance; sway variability was reduced during fixation of nearby targets, relative to sway during fixation of more distant targets. The postural change appeared to be functional in that the impact on gaze of a given motion of the body scales negatively with the distance of fixated targets (for detailed discussion, see Stoffregen et al., 2000; Stoffregen et al., 1999). We do not claim that the trial effects observed in this study have a functional relation to visual performance. However, the robust trial effects observed in this study suggest that it may be useful, in future research, to analyze for possible trial effects.

\section{GENERAL DISCUSSION}

In two experiments we found that postural motion of the body was reduced when participants were instructed to shift their gaze to follow a moving target, relative to body sway during fixation of stationary targets. Eye movements made when the eyes were closed had no effect on motion of the torso; thus, the influence of eye movements on torso motion appeared to be functionally related to visual performance. These are the major findings of this study and they provide support for the 
general hypothesis that body sway can be controlled, in part, so as to facilitate the achievement of suprapostural task goals. In addition, the angular displacement of visual targets was small enough so that required shifts in gaze could be accomplished without supporting head movements, and analysis of data on head motion confirmed that the head did not rotate differentially during viewing of moving and stationary targets. We discuss these results following.

\section{Reduced Sway During Eye Movements}

In this study, postural motion of the torso (and, in several cases, of the head) was reduced during visually guided eye movements relative to sway during fixation of a stationary target. Across two experiments and several manipulations, we never found an increase in sway during eye movements. Woollacott and Shumway-Cook (2002) argued that posture is not controlled in an automatic or reflexive manner. Our findings are compatible with their position. However, our findings do not fit comfortably with the most widely accepted interpretation of relations between the control of posture and suprapostural tasks. Many researchers have assumed that the control of posture relies on central cognitive processing resources (Dault et al., 2001; Lajoie et al., 1993; Maylor \& Wing, 1996; White et al., 1980; Woollacott \& Shumway-Cook, 2002; Yardley et al., 1999). An implication of this view is that the combination of postural control with simultaneous suprapostural tasks should produce competition for these central resources. Presumably, such a view leads to an interpretation of our experiments as combining a postural control task with an eye movement task, so that the two tasks would compete for central processing resources. Precisely this interpretation has been offered for a very similar situation studied by Hunter and Hoffman (2001). The competitive, dual-task view makes clear predictions about such situations. If the combined central processing demand of the two tasks exceeds the central processing resources that are available, then there should be a performance decrement in one or both of the combined tasks. If the combined central processing demand of the two tasks does not require more central processing resources than are available, then performance of the two tasks should be unaffected by the combination. This view has no apparent basis for predicting the result obtained in each of our experiments, in which postural sway was reduced when participants looked at a moving target, relative to sway during stationary fixation.

Our results also directly oppose predictions made by the saccadic suppression hypothesis (Oblak et al., 1985; White et al., 1980). Saccades may entail the suppression of visual information pickup, but any resulting loss of information about body sway clearly does not lead to an increase in postural motion.

The reduction in body sway while viewing a moving target is compatible with a different view of relations between postural control and suprapostural activity (Riccio \& Stoffregen, 1988). In general, we do not view postural control as being in competition for central processing resources with concurrent suprapostural activi- 
ties. This is because, for many activities, performance of suprapostural tasks may be influenced by postural motion. Because postural motion can influence the performance of suprapostural tasks, functional integration of postural control with suprapostural activity would be adaptive. In such circumstances, postural control would serve a dual function; on one hand, to avoid falling, and on the other hand, to optimize performance of suprapostural tasks (e.g., Massion, 1992). When the risk of falling is slight, as is generally the case for healthy adults and was certainly the case in our experiments, facilitation of suprapostural performance may play a stronger role in the organization of postural control. This view leads to the prediction that body sway may be reduced when such reductions can facilitate suprapostural performance (or, conversely, sway may be increased when such increases can facilitate suprapostural performance). When we view targets that are in motion, the success of eye movements (i.e., the maintenance of visual targets within the fovea) may be compromised by excessive body sway (among other things). Thus, one way to facilitate the viewing of moving targets might be to reduce sway. On this basis, we can predict that sway should be reduced when we look at moving targets, relative to sway during fixation of stationary targets. The results of each of our experiments are compatible with this hypothesis. ${ }^{6}$

Interpretations similar to ours have been offered by previous researchers, but on an ad hoc basis to explain unexpected findings. Oblak et al. (1985) found a reduction in mean radius of sway during visually guided eye movements, which contradicted their prediction of an increase in body sway during eye movements. In interpreting their results they suggested "the most logical conclusion is that the oculomotor system requires stabilization of posture to be able to hit the target light" (Oblak et al., 1985, p. 125). Similarly, Hunter and Hoffman (2001, p. 46) based their study on the a priori hypothesis of dual task competition for central processing resources but in light of their findings suggested a very different post hoc explanation: "A secondary task may, in effect, constrain the amount of acceptable postural sway." These post hoc explanations were made on an ad hoc basic (that is, without any motivation beyond the data at hand). The effects observed in this study were predicted a priori, and the predictions were derived from an established body of theory and research (Oullier, Bardy, Stoffregen, \& Bootsma, 2002; Riccio \& Stoffregen, 1988; Stoffregen et al., 2000; Stoffregen et al., 1999).

\footnotetext{
${ }^{6}$ Newell, van Emmerik, Lee, and Sprague (1993) reported an experiment demonstrating that variability may not be an appropriate dependent variable to use in operationalizing the concept of postural stability. We agree with their argument that variability is a poor measure of postural stability, and we have not used it in this way. We have used positional variability as a metric for the amplitude of body sway without interpreting any particular sway amplitude as being more or less stable. In part, this study (together with that of Stoffregen et al., 1999, and Stoffregen et al., 2000) can be interpreted as supporting the conclusions of Newell et al. Consistent with the position of Newell et al., we have not used positional variability as a measure of postural stability. Indeed, our position is that no measure of postural behavior, by itself, can be used to measure postural stability. In our view, the stability of postural control can be assessed only in the context of constraints imposed by suprapostural tasks.
} 
The results of this study, and those of Hunter and Hoffman, Kikukawa and Taguchi (1985), and Oblak et al., are consistent with the idea that eye movement control is not limited to the muscles that act on the eyes (e.g., Steinman et al., 1990), but can extend to muscles acting on the head and torso. The results are also consistent with J. J. Gibson's (1966) broader assertion that the entire body is part of the visual system. These results underline the fact that looking is an act; it is something that people do, rather than being merely a response to external stimulation. The act of looking (i.e., the stable control of the eyes relative to targets of interest) can be influenced by movements of a variety of body parts and, as indicated by our experiments, by movements of the entire body (see also Anderson et al., 2001).

\section{Postural Stabilization of Visually Guided Eye Movements}

The second major finding of our study is that eye movements did not lead to changes in body sway when the eyes were closed. This finding indicates that body sway was modulated to support visual performance, and not to support ocular control, per se. Presumably, eye movements require some level of effort whether the eyes are open or closed. Whether the same amount of effort would be needed for these two tasks is unclear. On one hand, it might be argued that visually guided eye movements require greater effort because the viewer must search out, pick up, and use information about the orientation of the eyes relative to the external environment, and because even minor errors in eye movements can lead to substantial degradation in visual performance. On the other hand, it might be argued that greater effort would be needed to move the eyes during eye closure because of the novelty of this task. Theories of automatic processing generally argue that tasks require more cognitive resources when they are novel than when they are well practiced (e.g., Kahneman, 1973). It is not clear how to determine, a priori, whether such theories would predict that more resources would be required to move the eyes when they were open or when they were closed. However, we might evaluate part of the argument by giving participants extensive training at eyes-closed eye movements. Practice should reduce the effort required for this type of eye movement. If the dual-task competition view were correct, there should be a decrease in body sway as participants became more practiced at eyes-closed eye movements. We would predict that this change would not occur. On the contrary, with long practice we would expect to see the effect of eyes-closed eye movements on head motion (the reduction in head movements during eyes-closed eye movements relative to head movements during stationary eye closure; Experiment 1) disappear as participants became more skilled at moving the eyes independent of body sway.

We have suggested that reductions in sway variability may have facilitated shifts in gaze in our experiments. We do not claim, however, that gaze stabilization would always imply reductions in sway. In some cases visual performance might be facilitated by increasing the overall magnitude of sway, provided that the increased mo- 
tion served to facilitate gaze. One recent study is consistent with this idea. Glasauer, Schneider, Jahn, Strupp, and Brandt (2005) measured sway and eye movements while standing participants looked at moving and stationary targets. Target motion consisted of sinusoidal horizontal oscillations at $0.33 \mathrm{~Hz}, 12^{\circ}$ amplitude. Stance was heel to toe. The results revealed an increase in RMS sway in the ML axis when moving targets were presented. This might be interpreted as conflicting with our finding that sway variability tended to be reduced when participants looked at moving targets, but such an interpretation can be questioned. Glasauer et al. did not evaluate the hypothesis that ML postural oscillations may have become coupled with the horizontal smooth pursuit movements used to track the visual targets. However, they presented median data for eye movements and for ML sway that strongly suggest that coupling occurred. Coupling of ML sway with horizontal eye movements may have been functional, that is, the coupling may have facilitated performance in the visual tracking task. Such an effect would be consistent with our general hypotheses. It would be interesting to examine links between gaze and postural control while varying stance (e.g., feet side by side vs. heel to toe) and while varying the type of eye movements required (saccades vs. smooth pursuit).

\section{The Role of Cognition}

In our experiments, was posture influenced by task-related constraints on the visual system, or by variations in the cognitive difficulty of tasks? The latter possibility might seem credible for tasks that require an obvious cognitive component, such as mental arithmetic (e.g., Yardley et al., 1999), or searching a text for target letters (e.g., Stoffregen et al., 2000), but it is less credible for the simple eye movements required in our experiments. With such simple eye movements it seems to us more reasonable to conclude that posture was influenced by constraints related to the visual tasks. The issue could be tested by varying the mental workload of visual tasks independently of their "ocular workload," that is, by independently varying levels of cognitive effort and ocular stability required for the performance of visual tasks.

\section{Shifting Gaze Without Head Rotation}

Our measurements of eye position confirm that participants moved their eyes, and that the amplitude of eye movements matched the amplitude of target displacement. The match between eye movement amplitude and target displacement fits well with our analysis of head rotation around the vertical axis, which showed that head rotation was not influenced by movement or stasis of the targets. Thus, we conclude not only that the postural effects observed in this study were related to movements of the eyes, but that they were not related to rotation of the head. We do not suggest that head movements are irrelevant to the influence of postural control of dynamic gaze. We eliminated head rotation solely because it would tend to alter motion of the torso, which would complicate our effort to understand rela- 
tions between eye movements and stance. When gaze shifts are large enough to elicit head rotation, we would expect that motion of the torso, head, and eyes are integrated to optimize visual performance.

\section{CONCLUSION}

Woollacott and Shumway-Cook (2002), defined postural control as "the control of the body's position in space for the purposes of balance and orientation" ( p. 1). Presumably, by orientation Woollacott and Shumway-Cook referred to the orientation of the body. Our research suggests that this definition may not fully capture the functional value of postural control. Our results suggest that one function of postural control is to stabilize the visual system so as to facilitate the accuracy of small changes in the direction of gaze. Beyond this our results, together with those of Stoffregen et al. (2000) and Stoffregen et al. (1999), suggest that there may be movement signatures of the difficulty of different suprapostural tasks.

We asked participants to move their eyes at constant amplitude and frequency. It would be interesting to vary the amplitude and/or frequency of eye movements in the context of postural sway. The integration of eye movements and body sway observed in this study might be influenced by the amplitude of eye movements (e.g., a negative relation between the amplitude of eye movements and the amplitude of sway), or by eye movement frequency (e.g., a negative relation between the frequency of eye movements and the amplitude of sway). Another area for future research concerns the dynamics of body sway and eye movements. Body sway is periodic motion and in our experiments eye movements were also periodic. Body sway is known to have characteristics of dynamical systems, such as stable coordination modes, sudden transitions between modes, critical slowing down, and hysteresis (e.g., Bardy, Marin, Stoffregen, \& Bootsma, 1999; Bardy, Oullier, Bootsma, \& Stoffregen, 2002; Oullier et al., 2002). When following the motion of oscillating targets, ocular control may also function as a dynamical system, in which case we might expect to observe coupling of the dynamics of the postural and ocular systems (e.g., phase locking). This study suggests that any dynamical coupling of these systems should be functional, that is, it should facilitate visual performance. Finally, our experiments were concerned exclusively with shifts in gaze achieved using saccadic eye movements. Future research should evaluate the hypothesis that body sway can be modulated to facilitate the performance of continuous changes in the direction of gaze (i.e., tracking eye movements).

\section{ACKNOWLEDGMENTS}

Randy J. Pagulayan is now at Microsoft Game Studios, Redmond, Washington.

Portions of these data were reported at the Eleventh International Conference on Perception and Action, Storrs, Connecticut, June, 2001. 
Preparation of this article was supported by Enactive Interfaces, a network of excellence (IST Contract No. 002114) of the Commission of the European Community; the National Science Foundation (BCS-0236627, INT-9603315); the University of Paris XI; and the Institut Universitaire de France. Experiment 1 was conducted as part of Randy J. Pagulayan's doctoral dissertation. We thank Michael A. Riley for comments on a draft of this article; Philip Hove, Omar Merhi, Valerie Neverman, Didier Casalta, Delphine Bernardin, and Olivier Oullier for their help with data collection and analysis; Dave Irwin for conversations and encouragement regarding this project; and Albert Gaudin, who provided the AMTI force plate.

\section{REFERENCES}

Abernethy, B. (1988). Dual-task methodology and motor skills research: Some applications and methodological constraints. Journal of Human Movement Studies, 14, 101-132.

American Psychological Association. (1992). Ethical principles of psychologists and code of conduct. Washington, DC: Author.

Anderson, D. I., Campos, J. J., Anderson, D. E., Thomas, T. D., Witherington, D. C., Uchiyama, I., et al. (2001). The flip side of perception-action coupling: Locomotor experience and the ontogeny of visual-postural coupling. Human Movement Science, 20, 461-487.

Bardy, B. G., Marin, L., Stoffregen, T. A., \& Bootsma, R. J. (1999). Postural coordination modes considered as emergent phenomena. Journal of Experimental Psychology: Human Perception and Performance, 25, 1284-1301.

Bardy, B. G., Oullier, O., Bootsma, R. J., \& Stoffregen, T. A. (2002). The dynamics of human postural transitions. Journal of Experimental Psychology: Human Perception and Performance, 28, 499-514.

Bernstein, N. (1996). On dexterity and its development. In M. L. Latash \& M. T. Turvey (Eds.), Dexterity and its development (pp. 3-244). Hillsdale, NJ: Lawrence Erlbaum Associates, Inc.

Berthoz, A. (1990). Reference frames for the perception and control of movement. In J. Paillard (Ed.), Brain and space. New York: Oxford University Press.

Cohen, J. D., MacWhinney, B., Flatt, M., \& Provost, J. (1993). PsyScope: A new graphic interactive environment for designing psychology experiments. Behavioral Research Methods, Instruments, $\mathcal{E}$ Computers, 25, 257-271.

Commissaris, D. A. C. M., \& Toussaint, H. M. (1997). Anticipatory postural adjustments in a bimanual, whole body lifting task with an object of known weight. Human Movement Science, 16, 407-431.

Cutting, J. E., Alliprandini, P. M. Z., \& Wang, R. F. (2000). Seeking one's heading through eye movements. Psychonomic Bulletin \& Review, 7, 490-498.

Dault, M. C., Geurts, A. C. H., Mulder, T. W., \& Duygens, J. (2001). Postural control and cognitive task performance in healthy participants while balancing on different support-surface configurations. Gait EO Posture, 14, 248-255.

Edwards, A. S. (1946). Body sway and vision. Journal of Experimental Psychology, 36, 526-535.

Friedli, W. G., Hallett, M., \& Simon, S. R. (1984). Postural adjustments associated with rapid voluntary arm movements, 1: Electromyographic data. Journal of Neurology, Neurosurgery, EF Psychiatry, 47, $611-622$.

Gibson, J. J. (1966). The senses considered as perceptual systems. Boston: Houghton Mifflin.

Giese, M. A., Dijkstra, T. M. H., Schöner, G., \& Gielen, C. C. A. M. (1996). Identification of the nonlinear state space dynamics of the action-perception cycle for visually induced postural sway. Biological Cybernetics, 74, 427-437. 
Glasauer, S., Schneider, E., Jahn, K., Strupp, M., \& Brandt, T. (2005). How the eyes move the body. Neurology, 65, 1292-1293.

Hallett, P. E. (1986). Eye movements. In K. R. Boff, L. Kaufman, \& J. P. Thomas (Eds.), Handbook of human perception and performance (Vol. 1, pp. 10-1-10-112). New York: Wiley.

Hunter, M. C., \& Hoffman, M. A. (2001). Postural control: Visual and cognitive manipulations. Gait $\mathcal{E}$ Posture, 13, 41-48.

Ivanenko, Y. P., Grasso, R., \& Lacquaniti, F. (1999). Effect of gaze on postural responses to neck proprioceptive and vestibular stimulation in humans. Journal of Physiology, 519, p. 301-314.

Jeka, J. (1995). Is servo theory the language of human postural control? Ecological Psychology, 7, 321-327.

Kahneman, D. (1973). Attention and effort. Englewood Cliffs, NJ: Prentice Hall.

Kerr, B., Condon, S. M., \& McDonald, L. A. (1985). Cognitive spatial processing and the regulation of posture. Journal of Experimental Psychology: Human Perception and Performance, 11, 617-622.

Kikukawa, M., \& Taguchi, K. (1985). Characteristics of body sway during saccadic eye movement in patients with peripheral vestibular disorders. In M. Igarashi and F. O. Black (Eds.), Vestibular and visual control on posture and locomotor equilibrium (pp. 355-359). New York: Karger.

Lajoie, Y., Teasdale, N., Bard, C., \& Fleury, M. (1993). Attentional demands for static and dynamic equilibrium. Experimental Brain Research, 97, 139-144.

Lee, W. A. (1980). Anticipatory control of postural and task muscles during rapid arm flexion. Journal of Motor Behavior, 12, 185-196.

Massion, J. (1992). Movement, posture and equilibrium: Interaction and coordination. Progress in Neurobiology, 38, 35-56.

Maylor, E. E., \& Wing, A. M. (1996). Age differences in postural stability are increased by additional cognitive demands. Journal of Gerontology, 51B, 143-154.

Nashner, L. M., \& McCollum, G. (1985). The organization of postural movements: A formal basis and experimental synthesis. The Behavioral and Brain Sciences, 8, 135-172.

Navon, D., \& Gopher, D. (1979). On the economy of the human-processing system. Psychological Review, $86,214-255$.

Newell, K. M. (1986). Constraints on the development of coordination. In M. G. Wade \& H. T. A. Whiting (Eds.), Motor development in children: Aspects of coordination and control (pp. 341-360). Boston: Martinus Nijhoff.

Newell, K. M., van Emmerik, R. E. A., Lee, D., \& Sprague, R. L. (1993). On postural stability and variability. Gait $\mathcal{E}$ Posture, 4, 225-230.

Oblak, B., Gregoric, M., \& Gyergyek, L. (1985). Effects of voluntary eye saccades on body sway. In M. Igarashi \& F. O. Black (Eds.), Vestibular and visual control on posture and locomotor equilibrium (pp. 122-126). New York: Karger.

Oullier, O., Bardy, B. G., Stoffregen, T. A., \& Bootsma, R. J. (2002). Postural coordination in looking and tracking tasks. Human Movement Science, 21, 147-167.

Pagulayan, R. J., Hayes, S., \& Stoffregen, T. A. (2001). Postural learning supports visual signal detection. In G. A. Burton \& R. C. Schmidt (Eds.), Studies in perception and action VI (pp. 193-196). Mahwah, NJ: Lawrence Erlbaum Associates, Inc.

Rayner, K. (1998). Eye movements in reading and information processing: 20 years of research. Psychological Bulletin, 124, 372-422.

Reed, E. S. (1988). Changing theories of postural development. In M. H. Woollacott \& A. Shumway-Cook (Eds.), Development of posture and gait (pp. 3-24). Columbia: University of South Carolina Press.

Riccio, G. E., \& Stoffregen, T. A. (1988). Affordances as constraints on the control of stance. Human Movement Science, 7, 265-300.

Riley, M. A., Mitra, S., Stoffregen, T. A., \& Turvey, M. T. (1997). Influences of body lean and vision on unperturbed postural sway. Motor Control, 1, 229-246.

Riley, M. A., Stoffregen, T. A., Grocki, M. J., \& Turvey, M. T. (1999). Postural stabilization of light touch. Human Movement Science, 18, 795-817. 
Skavenski, A. A., Hansen, R. M., Steinman, R. M., \& Winterson, B. J. (1979). Quality of retinal image stabilization during small natural and artificial body rotations in man. Vision Research, 19, 675-683.

Slijper, H., \& Latash, M. (2000). The effects of instability and additional hand support on anticipatory postural adjustments in leg, trunk, and arm muscles during standing. Experimental Brain Research, $135,81-93$.

Smart, L. J., Pagulayan, R., \& Stoffregen, T. A. (1998). Self-induced motion sickness in unperturbed stance. Brain Research Bulletin, 47, 449-457.

Steinman, R. M., \& Collewijn, H. (1980). Binocular retinal image motion during active head rotation. Vision Research, 20, 415-429.

Steinman, R. M., Kowler, E., \& Collewijn, H. (1990). New directions for oculomotor research. Vision Research, 30, 1845-1864.

Stoffregen, T. A., Pagulayan, R. J., Bardy, B. G., \& Hettinger, L. J. (2000). Modulating postural control to facilitate visual performance. Human Movement Science, 19, 203-220.

Stoffregen, T. A., Smart, L. J., Bardy, B. G., \& Pagulayan, R. J. (1999). Postural stabilization of looking. Journal of Experimental Psychology: Human Perception and Performance, 25, 1641-1658.

Teasdale, N., Bard, C., LaRue, J., \& Fleury, M. (1993). On the cognitive penetrability of posture control. Experimental Aging Research, 19, 1-13.

Wagner, M., Baird, J. C., \& Barbaresi, W. (1981). The locus of environmental attention. Journal of Environmental Psychology, 1, 195-201.

Warren, W. H. (1995). Self-motion: Visual perception and visual control. In W. Epstein and S. Rogers (Eds.), Perception of space and motion (pp. 262-326). New York: Academic.

Warren, W. H. (1998). The state of flow. In T. Watanabe (Ed.), High-level motion processing (pp. 315-358). Cambridge, MA: MIT Press.

Welch, R. B., Bridgeman, B., Williams, J. A., \& Semmler, R. (1998). Dual adaptation and adaptive generalization of the human vestibulo-ocular reflex. Perception EF Psychophysics, 60, 1415-1425.

White, K. D., Post, R. B., \& Leibowitz, H. W. (1980). Saccadic eye movements and body sway. Science, 208, 621-623.

Woollacott, M., \& Shumway-Cook, A. (2002). Attention and the control of posture and gait: A review of an emerging area of research. Gait $\mathcal{E}$ Posture, 16, 1-14.

Yardley, L., Gardner, M., Leadbetter, A., \& Lavie, N. (1999). Effect of articulatory and mental tasks on postural control. Neuroreport, 10, 1-5. 